\title{
Newer Innovations in Treatment of Retracted Nipple: Correspondence
}

\author{
Thirunavukkarasu Arun Babu • Pratap Kumar Patra
}

Received: 29 November 2012 / Accepted: 5 April 2013 / Published online: 25 July 2013

(C) Dr. K C Chaudhuri Foundation 2013

To the Editor: We read the article published by Jain $\mathrm{S}$ et al. entitled 'Newer Innovations in Treatment of Retracted Nipple' with interest [1]. We would like to congratulate the authors for taking up such a novel method and doing a commendable job.

Treating retracted nipple by vigorous sucking by husband is cheap and simple intervention which can be administered even in primary health care setup. However, there are some issues which require clarification. Authors mention that Group $\mathrm{C}$ cases were put on frequent and vigorous sucking at nipples by husbands. However, authors do not mention about the frequency, duration of intervention, gestational age at which it was started and whether it was evenly followed by all members in that group? How can authors be sure that cases in group $\mathrm{A}$ and $\mathrm{B}$ did not receive the intervention under study? Wouldn't it be a potential confounding factor in this study? Whether group A and B cases were clearly explained that they should not indulge in such acts. If these issues were not addressed, then the groups are not truly comparable and the results should be interpreted with a pinch of salt.

The disadvantages of this method include theoretical chances of transmitting infection to newborn due to poor oral hygiene in father, trauma to nipple and social acceptability. Social and cultural acceptability is an important factor restricting the use of this controversial method. Even doctors in our setup had mixed opinion regarding this. To know about the patient acceptability regarding this method in our setup, we did a questionnaire survey among antenatal and postnatal mothers in our hospital. Out of 121 respondents who completed the questionnaire, 16 (13.2\%) felt it was acceptable, 88 (72.7\%) felt it was not acceptable and 24 $(19.8 \%)$ did not have any comments. Though our observation completely contradicts the patient acceptability ratio $(79.7 \%)$ found in the study, it would be pertinent to know how the authors counselled their patients before including them into the study, any specific counselling methods used, problems faced and how they overcame those problems.

Though, this method sounds novel and has the potential for improving outcomes in nipple retraction, the patient acceptability seems to be a crucial factor. Therefore, proper counselling regarding nipple retraction, its consequences and lack of other treatment methods should be emphasized before introducing this concept to the mothers. Also, the superiority of this method over other existing methods should be confirmed by further trials.

\section{Reference}

1. Jain S, Jain A, Singh AK, Goswami D, Upadhyay AN, Negi $\mathrm{N}$. Newer innovations in treatment of retracted nipple. Indian $\mathrm{J}$ Pediatr. 2012; doi:10.1007s12098-012-0833-9 